Ilva Santana Santos Fonseca ${ }^{1}$

Tânia Maria de Araújo ${ }^{2}$

\section{Prevalência de transtornos mentais comuns em indus- triários da Bahia}

\author{
Prevalence of common mental disorders among industry \\ workers in Bahia, Brasil
}

\begin{abstract}
${ }^{1}$ Universidade Tiradentes. Aracaju, SE, Brasil.

${ }^{2}$ Núcleo de Epidemiologia, Departamento de Saúde da Universidade Estadual de Feira de Santana. Feira de Santana, BA, Brasil.

Contato:

Ilva Santana Santos Fonseca:

E-mail:

ilva_ss@hotmail.com

Artigo baseado na dissertação de mestrado de llva Santana Santos Fonseca intitulada Prevalência de transtornos mentais comuns em industriários da Bahia, 2011, defendida em 2011 na Universidade Estadual de Feira de Santana, Feira de Santana, Bahia, Brasil.
\end{abstract}

As autoras declaram que o trabalho não apresenta conflitos de interesses e não foi apresentado em reuniões científicas.

Trabalho não subvencionado.

Recebido: 10/07/2012

Revisado: 18/01/2013

Aprovado: 16/04/2013

\section{Resumo}

Introdução: há poucos estudos relacionando transtornos mentais comuns e trabalhadores da indústria. Objetivo: estimar a prevalência dos transtornos mentais comuns (TMC) entre os industriários da Bahia e verificar a sua associação com a ocupação. Métodos: estudo transversal, envolvendo 41.639 trabalhadores de empresas cadastradas na Relação Anual de Informações Sociais (RAIS) e no Sistema de Gerenciamento de Risco à Saúde na Indústria, do Serviço Social da Indústria (SESI). Para mensurar os TMC usou-se o General Health Questionnaire 12 (GHQ12) e para análise dos grupos ocupacionais (GG), a Classificação Brasileira de Ocupações (CBO). Resultados: a prevalência de TMC na população foi de 11,6\%. A associação dos TMC com os GG mostrou maior prevalência no GG4-trabalhadores de serviços administrativos $(13,2 \% ; R P=1,3 ; p<0,05)$. Após o ajuste pelas co-variáveis, os grupos GG1-dirigentes do poder público e de empresas $(\mathrm{RP}=2,4$, $\mathrm{p}=0,00)$ e GG2-profissionais das ciências e das artes $(\mathrm{RP}=2,3, \mathrm{p}=0,0)$ apresentaram as maiores prevalências em relação ao grupo de referência (GG3-técnicos de nível médio). Conclusão: o estudo permitiu traçar a distribuição dos TMC entre as ocupações dos industriários da Bahia, gerando informações que podem subsidiar a análise de variáveis sobre condições e organização do trabalho e a criação de estratégias que contribuam para a saúde mental dessa população.

Palavras-chave: transtornos mentais comuns; saúde do trabalhador; ocupações; GHQ.

\begin{abstract}
Background: there are few studies relating common mental disorders to industry workers. Objective: to estimate the prevalence of common mental disorders (CMD) among industry workers in Bahia, Brazil, and check association between disorders and occupations. Methods: a cross sectional study involving 41,639 workers from industries registered in the Brazilian Social Information Annual Report (RAIS) as well as in Management System on Health Risk in Industries from the Industry Social Service (SESI). To measure CMD we used the General Health Questionnaire GHQ-12, and to analyze occupational groups (GG), the Brazilian Classification of Occupations (CBO). Results: the CMD prevalence was $11.6 \%$. The CMD association with GG showed the highest prevalence in GG4-administrative service workers (13.2\%, PR=1.3, $p<0.05)$. After adjustment for covariates, groups GG1- heads of public service and enterprises $(R P=2.4, p=0.00)$ and GG2-science and art workers $(R P=2.3, p=0.0)$, had the highest prevalence when compared to the reference group (GG3-technicians with secondary school level). Conclusion: the study allowed tracking CMD among industry workers' occupations in Bahia, generating information that may not only subsidize analysis on labor conditions and organization, but also develop strategies to improve industry workers' mental health.
\end{abstract}

Keywords: common mental disorders; occupational health; occupations; GHQ. 


\section{Introdução}

Nas últimas décadas, o cenário mundial mudou consideravelmente refletindo transformações econômicas, culturais, sociais e políticas (FRANCO; DRUCK; SELIGMAN-SILVA, 2010). Estas modificações interferiram no âmbito da estrutura ocupacional, exigindo um novo olhar para o ambiente de trabalho e a vida dos trabalhadores.

Um dos setores ocupacionais que apresentou essas mudanças foi o industriário, que vem crescendo ao longo dos anos no Brasil. De 1996 a 2008, o número de indústrias no Brasil apresentou um aumento em torno de $260,0 \%$, concomitante com a desconcentração do setor da região sudeste do país para as demais regiões brasileiras (FEDERAÇÃO DAS INDÚSTRIAS DO ESTADO DA BAHIA, 2009).

A expansão industrial e as novas modalidades de gestão e produção neste setor têm instigado a realização de estudos, de diferentes áreas científicas, inclusive na área da saúde, que tem buscado relacionar o perfil de saúde-doença e as características das ocupações. Estas pesquisas têm relacionado as condições e a organização de trabalho, com diversas variáveis como comportamento de risco, auto-avaliação da saúde, estresse, prevalência de doenças físicas crônicas, como hipertensão arterial e psíquicas como transtornos mentais (NAHAS, 2001; HÖFELMANN; BLANK, 2007; FONSECA et al., 2008).

De acordo com a Organización Mundial de la Salud - OMS (2007), a saúde mental se define com um estado de bem-estar no qual o indivíduo é consciente de suas próprias capacidades, pode enfrentar as tensões da vida, trabalhar de forma produtiva e é capaz de contribuir para a sociedade.

Um projeto da OMS, iniciado em 2000, denominado Projeto Atlas, teve como objetivo cartografar a saúde mental de todo o mundo, e como um dos resultados verificou-se que uma em cada quatro pessoas no planeta terá algum transtorno mental ou neurológico em algum momento da vida (PAN AMERICAN HEALTH ORGANIZATION, 2001).

A definição de transtornos mentais comuns (TMC), adotada neste estudo, foi elaborada por Goldberg e Huxley (1992) e refere-se a uma expressão para designar transtornos mentais caracterizados por insônia, fadiga, irritabilidade, esquecimento, dificuldade de concentração e queixas somáticas (LUDERMIR; MELO-FILHO, 2002). Estudos mostram uma prevalência de TMC entre 7\% e 30\%, no mundo, e também revelam que cerca de 1/3 dos dias perdidos de trabalho, taxas de mortalidade mais elevadas e prejuízos nas funções sociais e físicas das pessoas es- tão relacionados a existência de transtornos mentais (LOPES; FAERSTEIN; CHOR, 2003).

Em levantamento na Biblioteca Virtual de Saúde (BIREME), em 2010, utilizando-se os descritores transtornos mentais comuns, saúde mental, industriários e indústria, encontraram-se duas referências de pesquisas sobre saúde mental e industriários. Benavides et al. (2002) estudaram fatores psicossociais em quatro empresas espanholas segundo características sociodemográficas. No Chile, Molina e Miasso (2008), verificaram a prevalência de consumo de benzodiazepínicos entre empregados de uma empresa privada.

No Brasil, estudos apontaram relação do trabalho com a morbidade psiquiátrica. Guimarães e Teixeira (2003) estimaram a prevalência de transtornos mentais comuns em operários de uma indústria de mineração de ferro em Minas Gerais, que trabalhavam em turnos alternados, e obtiveram uma prevalência de 16,6\%. Souza et al. (2010) relacionaram aspectos psicossociais do trabalho aos transtornos mentais comuns em trabalhadores de manutenção de equipamentos e linhas elétricas, na Bahia e em Sergipe, e observaram uma prevalência destes transtornos de 20,3\%.

Apesar dos estudos sobre transtornos mentais comuns em grupos ocupacionais no Brasil terem aumentado ao longo dos anos, ainda é restrito o número de pesquisas relacionando transtornos mentais comuns e os industriários. Assim, partindo-se desta necessidade, em um contexto caracterizado pelo mercado de trabalho cada vez mais exigente, mutante, competitivo, com demanda crescente de profissionais com diversas competências, as interferências na vida psicológica e social dos trabalhadores parecem ter sido intensificadas. A avaliação da situação de saúde mental dos trabalhadores nos mais diferentes contextos laborais pode levar ao redimensionamento de características do trabalho, podendo ser ferramenta importante na estruturação de ações para a proteção e promoção da saúde e da qualidade de vida no trabalho. Nesta perspectiva, este estudo objetivou estimar a prevalência dos transtornos mentais comuns entre os industriários do Estado da Bahia e avaliar a associação com a ocupação neste setor.

No presente trabalho, considerou-se a definição de industriário conforme a Classificação Brasileira de Ocupações (CBO), que compreende aquele trabalhador que, para desenvolver suas atividades principais, requer os conhecimentos e atividades necessários para produzir bens e serviços industriais (BRASIL, 2002). 


\section{Metodologia}

\section{Definição do tipo de estudo}

Tratou-se de um estudo epidemiológico, com delineamento transversal, realizado no Estado da Bahia com os industriários. A Bahia possui $417 \mathrm{mu}-$ nicípios, uma área geográfica de $564.692 .669 \mathrm{~km}^{2}$, população industrial estimada em 284.178 trabalhadores e 5.802 empresas industriais (FEDERAÇÃO DAS INDÚSTRIAS DO ESTADO DA BAHIA, 2010; INSTITUTO BRASILEIRO DE GEOGRAFIA E ESTATÍSTICA, 2010).

\section{População do estudo}

A população do estudo foi constituída pelo quantitativo de industriários cadastrados no "Diagnostico de saúde e estilo de vida do Trabalhador da indústria (DSEV), do ano de 2009, realizado pelo Serviço Social da Indústria (SESI), do Estado da Bahia.

O DSEV foi um diagnostico realizado pelo SESI entre os industriários do Brasil, a fim de se verificar as condições de saúde, trabalho, estilo de vida e qualidade de vida dos mesmos. Ele foi iniciado em 2007 através de um estudo piloto em uma empresa com 291 trabalhadores na cidade de Canoas / RS. Este estudo possibilitou o levantamento do perfil epidemiológico de fatores de risco para doenças não-transmissíveis destes trabalhadores.

Para serem incluídos no estudo no Estado da Bahia, os industriários deveriam estar em efetivo exercício profissional durante o período de coleta do DSEV, que foi entre janeiro e dezembro de 2009 e as empresas (318 no total) envolvidas deveriam estar cadastradas na Relação Anual de Informações Sociais (RAIS).

Foram consideradas indústrias, todas as empresas inseridas nos grandes grupos ocupacionais da CBO: GG6 a GG9, por se tratarem das industrias agropecuárias (GG6) e as industrias que fabricam bens, operam e mantem equipamentos (GG7 a GG9). Os demais grupos também foram analisados neste estudo, por terem sido inseridos durante a coleta de dados pelo SESI, já que dentro das empresas cadastradas, existiam funcionários desenvolvendo funções especificas dos demais grupos, contudo estavam lotados como industriários.

Além disso, durante a coleta, foram inelegíveis aqueles que estavam de licença médica, maternidade, de férias ou foram transferidos da empresa em analise, para outros estados ou outra unidade. Sendo assim, os elegíveis durante a pesquisa foram 59.477 trabalhadores, destes, 41.639 (70,0\%) foram incluí- dos na pesquisa, pois tiveram os dados dos questionários aplicados durante a coleta para levantamento dos dados, totalmente preenchidos no sistema de dados da mesma.

\section{Instrumento de pesquisa}

Para rastrear os transtornos mentais comuns utilizou-se o General Health Questionnaire (GHQ), instrumento elaborado por Goldberg e Huxley (1992) com a finalidade de detectar distúrbios psiquiátricos não psicóticos nos contextos comunitários e ocupacionais, a partir das respostas relatadas pelo entrevistado no momento da pesquisa. É um instrumento auto-aplicável para detectar distúrbios neuróticos, inicialmente foi composto por 60 itens, sendo posteriormente apresentado em versões resumidas, contendo 30, 20 e, por fim, 12 itens, sem ter sua confiabilidade comprometida (GOUVEIA et al., 2003).

A versão utilizada neste estudo foi a reduzida com 12 questões (GHQ-12), por escolha do grupo de trabalho do SESI durante o estudo piloto (PASQUALI et al., 1996; GOUVEIA et al., 2003).

O GHQ-12 é composto por cinco fatores: tensão ou estresse psíquico (destaca experiências de tensão, irritação, impaciência, cansaço e sobrecarga), desconfiança no próprio desempenho (expressa a consciência de ser capaz de desempenhar ou realizar as tarefas diárias de forma satisfatória), distúrbios do sono (relacionado a problemas com o sono, como insônias e pesadelos), distúrbios psicossomáticos (refere-se a problemas de ordem orgânica, como dores de cabeça, fraqueza e calafrios) e o fator geral para avaliar ausência de saúde mental (referente a severidade da falta de saúde mental) (PASQUALI et al., 1996).

Os itens de resposta do GHQ-12 consistem em uma escala de quatro pontos, do tipo Likert, que variam de "menos que o habitual" até "muito mais do que o habitual". De acordo com o método tradicional de Goldberg e Huxley, utilizado neste estudo, a apuração da resposta é realizada através do valor atribuído aos pontos dos itens, sendo dado o valor 0 (se o indivíduo escolher qualquer uma das duas primeiras alternativas da pergunta: 'melhor que o habitual' ou 'o mesmo de sempre') e 1 (se a escolha for pela terceira ou pela quarta alternativa: 'menos que o habitual' ou 'muito menos que o habitual') (PASQUALI et al., 1996).

A validação deste instrumento no Brasil, foi realizada por Gouveia et al. (2003), em uma amostra de 306 adultos da população de João Pessoa, Paraíba, onde a confiabilidade do instrumento foi verificada através do coeficiente $\alpha$ de Cronbach, obtendo-se 0,84 . 


\section{Definição das variáveis de estudo}

\section{Variável resposta: transtornos mentais comuns}

Os Transtornos Mentais Comuns (TMC) foram avaliados através do General Health Questionnaire (GHQ-12) e para verificar o nível de suspeição de TMC, durante o estudo, realizou-se a análise de desempenho do GHQ-12 através da Análise da Curva ROC (Receiver Operating Characteristic), a fim de se definir os níveis de sensibilidade e de especificidade com melhor desempenho de acordo com o método de pontuação do questionário da presente pesquisa.

O perfil sintomático neste estudo foi definido através da relação com o percentil correspondente na Tabela de Normas da Amostra de Padronização do Manual Técnico de Aplicação do GHQ - adaptação brasileira (1996), que se adequou ao percentil 90, tendo como ponto de corte a pontuação equivalente a 3 ou mais (PASQUALI et al., 1996).

O ponto de corte de melhor desempenho encontrado foi 3 ou mais, com uma sensibilidade de 99,4\% e especificidade de 96,1\%, e área sob a curva ROC de 0,987 com um desvio padrão de 0,001 e intervalo de $95 \%$ de confiança variando de 0,985 a 0,989, apontando assim um nível excelente de discriminação entre casos e não casos.

\section{Variável preditora: grupos ocupacionais segundo CBO}

Neste estudo, os grupos ocupacionais foram definidos de acordo com a Classificação Brasileira de Ocupações - CBO (BRASIL, 2002). De acordo com a CBO, existem 10 grandes grupos de ocupações, 47 subgrupos principais (SGP), que são agrupamentos mais restritos que os grandes grupos, 192 subgrupos (SG), também denominados de grupos primários, grupos unitários e famílias ocupacionais, que reúnem ocupações que apresentam estreito parentesco tanto em relação à natureza de trabalho quanto aos níveis de qualificações exigidos, 596 grupos de base ou famílias ocupacionais (GB), onde se agrupam 2.422 ocupações.

Os dez grandes grupos ocupacionais segundo a CBO são:

GG0

GG1

GG2

GG3

GG4

GG5

GG6
Forças Armadas, Policiais e Bombeiros Militares

Membros superiores do poder público, dirigentes de organizações de interesse público e de empresas e gerentes

Profissionais das ciências e das artes

Técnicos de nível médio

Trabalhadores de serviços administrativos

Trabalhadores dos serviços, vendedores do comércio em lojas e mercados

Trabalhadores agropecuários, florestais, da caça e pesca
Trabalhadores da produção de bens e serviços industriais (artesanais)

GG8

Trabalhadores da produção de bens e serviços industriais (produção)

GG9

Trabalhadores de manutenção e reparação

As ocupações do setor industrial estudadas foram agrupadas nesses 10 grandes grupos ocupacionais, conforme descrito anteriormente.

\section{Co-variáveis:}

Além dos 10 grandes grupos ocupacionais foram analisadas variáveis sociodemográficas (sexo; estado civil - casado / união estável, solteiro, divorciado/viúvo; faixa etária (até 25 anos, 26 a 35 anos, 36 a 45 anos, 46 a 60 anos e acima de 60 anos); raça / cor da pele (branco, pardo, preto, indígena, amarelo); escolaridade (ensinos fundamental completo, médio completo, superior completo e pós-graduação concluída); renda mensal (até 1 salário mínimo, entre 3 e 5 salários, 5 e 10 e acima de 10 salários). Foram avaliadas também variáveis ocupacionais: carga horária de trabalho semanal (até 40 horas e acima de 40 horas); dias de trabalho na semana (até 6 dias e acima de 6 dias); o turno de trabalho é fixo e se trabalha algum dia à noite. A variável "turno de trabalho é fixo", diz respeito ao turno ser apenas pela manhã, tarde ou noite, de forma invariável, já a variável "se trabalha algum dia à noite", busca informações sobre, se o trabalhador, não tem seu turno fixo de trabalho e, alguma vez durante sua escala de trabalho, atua a noite.

\section{Análise dos dados}

Foi conduzida análise descritiva para conhecimento do perfil sociodemográfico da população (sexo, estado civil, faixa etária, cor/raça, escolaridade e renda mensal) e características do trabalho (carga horária de trabalho diária, dias de trabalho semanal, turno de trabalho é fixo, se trabalha algum dia à noite) da população estudada, além da distribuição das proporções de trabalhadores entre os grandes grupos ocupacionais da Classificação Brasileira de Ocupações.

Após a análise descritiva, conduziu-se a análise de associação bruta para avaliar a relação entre os grupos ocupacionais e os transtornos mentais comuns. Foram calculadas as prevalências e as razões de prevalência. Para avaliação da associação entre as variáveis, utilizou-se o teste qui-quadrado de Wald, adotando-se $p$ valor $\leq$ 0,05 para associações estatisticamente significantes.

Para verificar a interação das variáveis do estudo, fez-se uso da modelagem de dados estatístico através da Análise de Regressão Logística Multivariada 
(ARLM), que é adequada para análises onde a variável resposta é dicotômica. Através deste modelo, buscou-se verificar o efeito dos grupos ocupacionais sobre os transtornos mentais comuns, considerando o efeito das demais variáveis analisadas (sociodemográficas e ocupacionais) (HOSMER; LEMESHOW, 2000; HOSMER; LEMESHOW; MAY, 2008).

A análise de regressão logística produz como medida de associação a odds ratio, assim, baseada na estimativa das probabilidades de transtornos mentais comuns segundo os grandes grupos ocupacionais, converteu-se a odds ratio em razão de prevalência. Neste caso, o conceito de ajuste é tido com predição condicional, onde a média dos ajustantes (co-variáveis) é usada na estimação da prevalência em cada grupo (COUTINHO; SCAZUFCA; MENEZES, 2008).

A escolha das variáveis preditoras obedeceu apresentação do valor de $p<0,25$ no teste da razão de verossimilhança (definida como a probabilidade de se observar os dados efetivamente observados, em função do modelo probabilístico), o que permite a entrada no modelo de variáveis que se tornam significativas quando em conjunto com os demais preditores. Na análise, as variáveis que apresentaram $p$ $>$ 0,10 no teste estatístico de Wald foram excluídas uma por vez (KLEINBAUM; KLEIN, 2002).

A bondade do ajuste do modelo foi analisada através da comparação da log-verossimilhança do modelo completo com o modelo apenas com o intercepto.
Os dados foram armazenados no Sistema de Gerenciamento de Risco Saúde na Indústria (SGRSI) e depois exportados para o SPSS (Statical Package for Social Sciences), na versão 10.0 for Windows para análise dos dados. Foram também utilizados para análise o Epi Info versão 3.4.1 e o R versão 2.11.1.

\section{Aspectos éticos}

Esta pesquisa foi aprovada pelo Comitê de Ética em Pesquisa da Universidade Estadual de Feira de Santana, sob o protocolo 106/2010, seguindo as especificações da Resolução 196/96 do Conselho Nacional de Saúde/ Ministério da Saúde, e de acordo com os quatros referenciais básicos da Bioética: a autonomia, maleficência, beneficência e justiça (BRASIL, 1996).

\section{Resultados}

A Tabela 1 descreve as características sociodemográficas da população de industriários. Os dados do estudo revelaram uma população predominantemente masculina (82,9\%), na faixa etária de 26 a 35 anos (41,0\%) (média de 35,3 \pm 10,0 anos), da cor parda (50,8\%), casados / união estável (57,8\%), com renda média mensal de 1 a 3 salários mínimos (76,8\%) e com nível de escolaridade no ensino médio completo $(47,8 \%)$.

Tabela 1 Caracterização da população de industriários segundo variáveis sociodemográficas e de trabalho. Bahia, 2009

\begin{tabular}{lcc}
\multicolumn{1}{c}{ Variáveis (N) } & $n$ & $\%$ \\
\hline Sexo (41418) & 34348 & 82,9 \\
Masculino & 7070 & 17,1 \\
Feminino & & \\
Faixa etária (41625) & 6792 & 16,3 \\
Até 25 anos & 17079 & 41,0 \\
De 26 a 35 anos & 10101 & 24,3 \\
De 36 a 45 anos & 6854 & 16,5 \\
De 46 a 60 anos & 799 & 1,9 \\
Acima de 60 anos & & \\
Raça / cor da pele (39788) & & \\
Branco & 6293 & 15,8 \\
Preto & 11724 & 29,5 \\
Amarelo & 926 & 2,3 \\
Pardo & 20226 & 50,8 \\
Indígena & 619 & 1,6 \\
\hline
\end{tabular}


(...) Tabela 1 Caracterização da população de industriários segundo variáveis sociodemográficas e de trabalho. Bahia, 2009

\begin{tabular}{lcc}
\multicolumn{1}{c}{ Variáveis (N) } & $n$ & $\%$ \\
\hline Estado civil (40993) & 23709 & 57,8 \\
$\quad$ Casado/união estável & 15946 & 38,9 \\
$\quad$ Solteiro & 1338 & 3,3 \\
$\quad$ Divorciado/Viúvo & & \\
& & \\
Renda média (40373) & 3009 & 7,5 \\
Até 1 Salário Mínimo (SM) & 31060 & 76,8 \\
1 SM à 3 SM & 3583 & 8,9 \\
Acima de 3 SM a 5 SM & 1849 & 4,6 \\
Acima de 5 SM a 10 SM & 872 & 2,2 \\
Acima de 10 SM & & \\
& & 1,2 \\
Escolaridade (41222) & 509 & 36,1 \\
Nunca estudou & 14877 & 47,8 \\
Ensino fundamental completo & 19673 & 12,1 \\
Ensino médio completo & 4999 & 2,8 \\
Ensino superior completo & 1164 & \\
Pós-graduação concluída & & \\
\hline
\end{tabular}

Houve perdas nas variáveis: sexo (220), faixa etária (13), raça/cor da pele (1850), estado civil (645), renda média mensal (1265), escolaridade (416).

Fonte: Diagnóstico de Saúde e Estilo de Vida (SESI/BAHIA).

As características do trabalho estão apresentadas na Tabela 2. Houve predomínio dos indivíduos com carga horária de trabalho semanal acima de 40 horas $(61,3 \%)$, trabalhando até seis dias por semana $(88,0 \%)$, em um mesmo horário todos os dias $(87,8 \%)$ e sem trabalhar à noite pelo menos um dia na semana $(78,8 \%)$.

Ao se distribuir a população segundo os grandes grupos da Classificação Brasileira de Ocupações (CBO), o grupo GG7 (trabalhadores da produção de bens e serviços industriais) concentrou a maioria dos trabalhadores (59,1\%), seguido pelo GG4 (trabalhadores de serviços administrativos) (10,8\%) (Tabela 2).

A prevalência global de transtornos mentais comuns foi de $11,6 \%$.

A associação das características sociodemográficas com os transtornos mentais revelou que as menores prevalências foram observadas entre os indivíduos mais velhos, sendo mais baixas ainda entre aqueles com mais de 60 anos, e com nível de escolaridade mais baixo. Os indivíduos homens e com renda acima de 5 salários mínimos e que nunca estudaram, apresentaram prevalências maiores. As prevalências de TMC entre os estratos na variável cor / raça, foram praticamente iguais, apresentando significância apenas entre pretos e pardos. (Tabela 3).
Com relação às características ocupacionais, as prevalências se mantiveram quase as mesmas entre os estratos das variáveis analisadas, apresentando significância em algumas delas. Aqueles trabalhadores que trabalhavam acima de 40 horas semanais e acima de 6 dias por semana, mostraram 10,0\% a mais de prevalência de transtornos mentais comuns, comparados aos que tinham carga horária menor que 40 horas e até 6 dias de trabalho na semana. Os indivíduos que trabalhavam pelo menos um dia na semana à noite tiveram $20 \%$ a mais de prevalência de TMC em relação aqueles que não trabalhavam a noite.

O grupo ocupacional, com maior prevalência de TMC foi o GG4 $(13,2 \%)$ (trabalhadores de serviços administrativos) e com menor o GG3 $(5,0 \%)$ (técnicos de nível médio), o qual foi tomado como grupo não exposto, devido não haver justificativa teórica na literatura que defina, entre os grandes grupos ocupacionais da CBO, o grupo de referência para estudos sobre transtornos mentais comuns (Tabela 4).

Na Tabela 5, são descritos os resultados da análise multivariada, realizada com o objetivo de verificar o efeito que as co-variáveis exercem sobre relação da prevalência dos transtornos mentais com os grupos ocupacionais. 
Tabela 2 Distribuição da população de industriários segundo características do trabalho. Bahia, 2009

\begin{tabular}{|c|c|c|}
\hline Variável (N) & $n$ & $\%$ \\
\hline \multicolumn{3}{|l|}{ Carga horária semanal (41223)* } \\
\hline Até 40 horas & 25289 & 61,3 \\
\hline Acima de 40 horas & 15934 & 38,7 \\
\hline \multicolumn{3}{|l|}{ Dias de trabalho semanal $(41144)^{*}$} \\
\hline Até 6 dias & 36189 & 88,0 \\
\hline Acima de 6 dias & 4955 & 12,0 \\
\hline \multicolumn{3}{|l|}{ O turno de trabalho é fixo $(41158)^{*}$} \\
\hline Sim & 36138 & 87,8 \\
\hline Não & 5020 & 12,2 \\
\hline \multicolumn{3}{|l|}{ Trabalha algum dia a noite (40493) } \\
\hline Sim & 8565 & 21,2 \\
\hline Não & 31928 & 78,8 \\
\hline \multicolumn{3}{|l|}{ Grandes grupos ocupacionais (39104) } \\
\hline GG1 - organizações de interesse público e de empresas e gerentes & 539 & 1,4 \\
\hline GG2 - Profissionais das ciências e das artes & 1041 & 2,7 \\
\hline GG3 - Técnicos de nível médio & 2294 & 5,9 \\
\hline GG4 - Trabalhadores de serviços administrativos & 4215 & 10,8 \\
\hline GG5 - Trabalhadores dos serviços, vendedores do comércio em lojas e mercados & 1754 & 4,5 \\
\hline GG6 - Trabalhadores agropecuários, florestais, da caça e pesca & 2790 & 7,1 \\
\hline GG7 - Trabalhadores da produção de bens e serviços industriais & 23123 & 59,1 \\
\hline GG8 - trabalhadores da produção de bens e serviços industriais & 2162 & 5,5 \\
\hline GG9 - Trabalhadores de manutenção e reparação & 1186 & 3,0 \\
\hline
\end{tabular}

* Houve perdas: 495 para dias de trabalho, 416 para carga horária, 481 para trabalha no mesmo horário, 1146 para trabalha à noite, 415 para carga horária de trabalho e 494 para dias de trabalho semanal.

Fonte: Diagnóstico de Saúde e Estilo de Vida (SESI/BAHIA)

Tabela 3 Prevalência de transtornos mentais comuns entre os industriários segundo variáveis sociodemográficas. Bahia, 2009

\begin{tabular}{|c|c|c|c|c|}
\hline \multirow[b]{2}{*}{ Variáveis $(\mathrm{N})^{*}$} & \multicolumn{2}{|c|}{ TMC } & \multirow[b]{2}{*}{$\mathrm{RP}^{* * * *}$} & \multirow[b]{2}{*}{ Valor de $\mathrm{p}$} \\
\hline & $\mathrm{n}$ & $\%$ & & \\
\hline \multicolumn{5}{|l|}{ Sexo (41161) } \\
\hline Masculino & 4081 & 12,0 & - & - \\
\hline Feminino & 705 & 10,1 & 0,8 & 0,00 \\
\hline \multicolumn{5}{|l|}{ Faixa etária (41367) } \\
\hline Até 25 anos & 855 & 12,6 & - & - \\
\hline 26 a 35 anos & 2067 & 12,6 & 1,0 & 0,33 \\
\hline 36 a 45 anos & 1164 & 11,6 & 0,9 & 0,04 \\
\hline 46 a 60 anos & 658 & 9,7 & 0,8 & 0,00 \\
\hline Acima de 60 anos & 60 & 7,5 & 0,6 & 0,00 \\
\hline
\end{tabular}


(...) Tabela 3 Prevalência de transtornos mentais comuns entre os industriários segundo variáveis sociodemográficas. Bahia, 2009

\begin{tabular}{|c|c|c|c|c|}
\hline \multirow{2}{*}{ Variáveis $(\mathrm{N})^{*}$} & \multicolumn{2}{|c|}{ TMC } & \multirow{2}{*}{$\mathrm{RP}^{* * *}$} & \multirow{2}{*}{ Valor de $\mathrm{p}$} \\
\hline & $\mathrm{n}$ & $\%$ & & \\
\hline \multicolumn{5}{|l|}{ Cor/raça (39663) } \\
\hline Branco & 809 & 12,9 & - & - \\
\hline Preto & 1341 & 11,5 & 0,9 & 0,00 \\
\hline Amarelo & 130 & 14,1 & 1,1 & 0,30 \\
\hline Pardo & 2240 & 11,1 & 0,9 & 0,00 \\
\hline Indígena & 81 & 13,2 & 1,0 & 0,83 \\
\hline \multicolumn{5}{|l|}{ Estado civil (40855) } \\
\hline Casado/vive junto & 2432 & 10,3 & - & - \\
\hline Solteiro & 2080 & 13,1 & 1,3 & 0,00 \\
\hline Divorciado/viúvo & 219 & 16,4 & 1,6 & 0,00 \\
\hline \multicolumn{5}{|l|}{ Renda mensal (40242) } \\
\hline Até $1 \mathrm{SM}$ & 497 & 16,6 & 1,0 & 0,92 \\
\hline 1 SM à 3 SM & 3173 & 10,2 & 0,6 & 0,00 \\
\hline $3 \mathrm{SM}$ a $5 \mathrm{SM}$ & 503 & 14,1 & 0,8 & 0,04 \\
\hline $5 \mathrm{SM}$ a $10 \mathrm{SM}$ & 329 & 17,9 & 1,1 & 0,47 \\
\hline Acima de 10 SM & 146 & 16,8 & - & - \\
\hline \multicolumn{5}{|l|}{ Escolaridade (41080) } \\
\hline Nunca estudou & 30 & 5,9 & 0,3 & 0,00 \\
\hline Nível fundamental & 1085 & 7,3 & 0,4 & 0,00 \\
\hline Nível médio & 2429 & 12,4 & 0,6 & 0,00 \\
\hline Nível superior & 981 & 19,7 & 1,0 & 0,52 \\
\hline Pós-graduação & 238 & 20,6 & - & - \\
\hline
\end{tabular}

* Houve perdas nas variáveis: sexo (478), faixa etária (272), raça/cor da pele (1976), estado civil (784), renda média mensal (1397), escolaridade (559).

***:Razão de Prevalências - grupos de referência: sexo (masculino); faixa etária (até 25 anos); cor/raça (branco); estado civil (casado/vive junto); renda mensal (acima de 10 SM); escolaridade (pós-graduação).

Fonte: Diagnóstico de Saúde e Estilo de Vida (SESI/BAHIA)

Tabela 4 Prevalência de transtornos mentais comuns entre os industriários segundo variáveis ocupacionais. Bahia, 2009

\begin{tabular}{|c|c|c|c|c|}
\hline \multirow{2}{*}{ Variáveis $(\mathrm{N})^{*}$} & \multicolumn{2}{|c|}{$T M C$} & \multirow{2}{*}{$R P^{* * *}$} & \multirow{2}{*}{ Valor de $p$} \\
\hline & $n$ & $\%$ & & \\
\hline \multicolumn{5}{|l|}{ Carga horária semanal (41091) } \\
\hline Até 40 horas & 2851 & 11,3 & - & - \\
\hline Acima de 40 horas & 1914 & 12,0 & 1,1 & 0,02 \\
\hline \multicolumn{5}{|l|}{ Dias de trabalho semanais (41054) } \\
\hline Até 6 dias & 4115 & 11,4 & - & - \\
\hline Acima de 6 dias & 643 & 13,0 & 1,1 & 0,00 \\
\hline \multicolumn{5}{|l|}{ O turno de trabalho é fixo (41024) } \\
\hline Sim & 4179 & 11,6 & - & - \\
\hline Não & 566 & 11,3 & 1,0 & 0,56 \\
\hline \multicolumn{5}{|l|}{ Trabalha algum dia à noite (40364) } \\
\hline Sim & 1121 & 13,1 & 1,2 & 0,00 \\
\hline Não & 3518 & 11,8 & - & - \\
\hline
\end{tabular}


(...) Tabela 4 Prevalência de transtornos mentais comuns entre os industriários segundo variáveis ocupacionais. Bahia, 2009

\begin{tabular}{|c|c|c|c|c|}
\hline \multirow{2}{*}{ Variáveis $(\mathrm{N})^{*}$} & \multicolumn{2}{|c|}{$T M C$} & \multirow{2}{*}{$R P^{\text {*** }}$} & \multirow[b]{2}{*}{ Valor de $p$} \\
\hline & $n$ & $\%$ & & \\
\hline \multicolumn{5}{|l|}{ Grandes grupos ocupacionais (38854) } \\
\hline $\begin{array}{l}\text { GG1 - organizações de interesse } \\
\text { público e de empresas e gerentes }\end{array}$ & 64 & 11,9 & 2,4 & 0,00 \\
\hline GG2 - Profissionais das ciências e das artes & 106 & 10,2 & 2,0 & 0,00 \\
\hline GG3*楼 - Técnicos de nível médio & 115 & 5,0 & - & - \\
\hline GG4 - Trabalhadores de serviços administrativos & 553 & 13,2 & 2,6 & 0,00 \\
\hline $\begin{array}{l}\text { GG5 - Trabalhadores dos serviços, vendedores do comércio } \\
\text { em lojas e mercados }\end{array}$ & 204 & 11,7 & 2,3 & 0,00 \\
\hline $\begin{array}{l}\text { GG6 - Trabalhadores agropecuários, florestais, da caça e } \\
\text { pesca }\end{array}$ & 343 & 12,3 & 2,5 & 0,00 \\
\hline $\begin{array}{l}\text { GG7 - Trabalhadores da produção } \\
\text { de bens e serviços industriais }\end{array}$ & 2778 & 12,1 & 2,4 & 0,00 \\
\hline $\begin{array}{l}\text { GG8 - trabalhadores da produção } \\
\text { de bens e serviços industriais }\end{array}$ & 221 & 10,3 & 2,1 & 0,00 \\
\hline GG9 - Trabalhadores de manuten-ção e reparação & 128 & 10,9 & 2,2 & 0,00 \\
\hline \multicolumn{5}{|c|}{ *Houve perdas nas variáveis: carga horária de trabalho (548) e dias de trabalho semanal (585). } \\
\hline \multicolumn{5}{|c|}{$\begin{array}{l}\text { Razão de Prevalências - grupos de referência: carga horária semanal (até } 40 \text { horas); dias de trabalho semanais (até } 6 \text { dias); o turno } \\
\text { de trabalho é fixo (sim); trabalha algum dia à noite (não). }\end{array}$} \\
\hline \multicolumn{5}{|c|}{$\begin{array}{l}\text { Utilizou-se o grupo GG3 como referência, por não haver na literatura justificativa teórica para a definição de um grupo de referên- } \\
\text { cia, além deste ter apresentado a menor prevalência de TMC. }\end{array}$} \\
\hline Fonte: Diagnóstico de Saúde e Estilo de Vida (SESI/BAHIA) & & & & \\
\hline
\end{tabular}

Tabela 5 Análise da regressão logística multivariada para transtornos mentais comuns em industriários. Bahia, 2009

\begin{tabular}{|c|c|c|c|c|}
\hline \multirow{3}{*}{ Variáveis (N) } & \multicolumn{4}{|c|}{ Razão de Prevalências (RP) } \\
\hline & \multicolumn{2}{|c|}{$R P_{\text {bruta }}$} & \multicolumn{2}{|c|}{$R P_{\text {ajustada }}$} \\
\hline & $R P$ & Valor de $p$ & $R P$ & Valor de $p$ \\
\hline GG1 - organizações de interesse público e de empresas e gerentes & 2,4 & 0,00 & 2,4 & 0,00 \\
\hline GG2 - Profissionais das ciências e das artes & 2,0 & 0,00 & 2,3 & 0,00 \\
\hline GG3 - Técnicos de nível médio & - & - & - & - \\
\hline GG4 - Trabalhadores de serviços administrativos & 2,6 & 0,00 & 2,1 & 0,00 \\
\hline $\begin{array}{l}\text { GG5 - Trabalhadores dos serviços, vendedores do comércio em lojas } \\
\text { e mercados }\end{array}$ & 2,3 & 0,00 & 1,6 & 0,00 \\
\hline GG6 - Trabalhadores agropecuários, florestais, da caça e pesca & 2,5 & 0,00 & 1,6 & 0,00 \\
\hline GG7 - Trabalhadores da produção de bens e serviços industriais & 2,4 & 0,00 & 1,8 & 0,00 \\
\hline GG8 - trabalhadores da produção de bens e serviços industriais & 2,1 & 0,00 & 1,7 & 0,00 \\
\hline GG9 - Trabalhadores de manutenção e reparação & 2,2 & 0,00 & 1,6 & 0,00 \\
\hline
\end{tabular}

A associação foi ajustada pelas seguintes variáveis, de acordo com o $p_{\text {valor }}<0,25$ do teste de razão de verossimilhança e para a modificação de efeito, $p_{\text {valor }}<0,10$ : Sexo, faixa etária, renda mensal, estado civil, carga horária, dias de trabalho semanais, trabalha no mesmo horário, e trabalha a noite (todas com $p=0,00)$ e escolaridade $(p=0,01)$. Bondade do ajuste $\left(X^{2}=814,71 ; p_{\text {valor }}=0,00\right)$ 
Todas as co-variáveis tiveram o valor de $p$ do teste de razão de verossimilhança abaixo de 0,25 e foram introduzidas no modelo de regressão. Na análise de ajustamento do modelo, a variável cor / raça ( $p$ $=0,11$ ), que apresentou $p>0,10$ no teste estatístico de Wald foi excluída do modelo (Tabela 5).

Após o ajuste do modelo, houve modificação nas prevalências de TMC entre os grupos demonstrando interação com as co-variáveis. As prevalências diminuíram em alguns grupos (com diminuições que variaram de $15,0 \%$, entre os profissionais das ciências e das artes - Grande Grupo 2 (GG2) -, a 36,0\%, entre os trabalhadores dos serviços, vendedores do comércio em lojas e mercados - Grande Grupo 5 (GG5) -, que na associação bruta apresentou maior prevalência de TMC) e aumentaram em outros (de 23,5\% no grupo dos trabalhadores da produção de bens e serviços industriais - Grande Grupo 8 (GG8) - até $56,2 \%$, entre trabalhadores agropecuários, florestais, da caça e pesca - Grande Grupo 6 (GG6), revelando uma provável ação de amenização ou de aumento destas co-variáveis sobre a associação dos grupos ocupacionais com os transtornos mentais comuns. Os grupos que apresentaram as razões de prevalências maiores em relação ao grupo de referência, após o ajustamento, foram os grupos dos trabalhadores das organizações de interesse público e de empresas e gerentes (GG1) e profissionais das ciências e das artes (GG2). O modelo se mostrou bem ajustado ( $\mathrm{X}^{2}$ $\left.=814,71 ; p_{\text {valor }}=0,00\right)($ Tabela 5).

\section{Discussão}

A prevalência de TMC na população estudada foi de 11,6\%. Esta prevalência mostrou-se baixa quando comparada às encontradas em outros estudos entre grupos ocupacionais - como operários de empresa de mineração $(16,6 \%)$ e de eletricistas $(20,3 \%)$. - Contudo, apesar do GHQ ser um instrumento apenas de rastreabilidade e não de diagnóstico de transtornos mentais comuns, esta prevalência revela uma situação onde ações preventivas devem ser aplicadas para o não agravamento deste quadro e/ou para possível diminuição desta na população do estudo (GUIMARÃES; TEIXEIRA, 2003; SOUZA et al., 2010).

Ao se verificar a associação da prevalência de transtornos mentais comuns com as características sociodemográficas dos industriários, observou-se que as associações com algumas destas características (sexo, faixa etária, estado civil, renda mensal e escolaridade) foram estatisticamente significantes. Nestas associações, , observou-se que as maiores prevalências foram entre os homens, mais jovens, com rendas médias mensais elevadas e com alto grau de escolaridade. Diante destes achados, é relevante analisar o cenário que estes trabalhadores estavam inseridos no período da pesquisa e que pode ter proporcionado estas associações.

O setor industrial no Brasil, devido ao grande crescimento que se observou ao longo dos anos, atraiu grandes indústrias internacionais, além de fazer com que as nacionais se preparassem para concorrer com o mercado externo. Isto fez com que o número de exigências na qualidade dos processos de trabalho, alinhado a dependência do mercado internacional, requeressem mais do trabalhador, que neste setor da economia brasileira, é composta majoritariamente pela população masculina (LINHART, 2009; BOUYER, 2010).

Devido a estreita relação com o mercado externo, o ramo industrial brasileiro está sujeito as instabilidades econômicas mundiais que podem refletir no mercado interno e, conseqüentemente, no ambiente de trabalho, como a que se iniciou em 2007 e perdurou até 2009 (POCHMANN, 2009; SPITZ, 2010).

Em 2007, eclodiu a crise financeira mundial nos Estados Unidos, que afetou o ramo industrial diretamente, porém as indústrias brasileiras sentiram a crise no segundo semestre de 2008, prolongando-se até o ano de 2009. Neste período, diversas indústrias no mundo e no Brasil, anunciaram diversas demissões, férias coletivas, diminuição na produção, redução nos custos, e este cenário afetou diretamente a mão-de-obra industrial, que viu na crise o aumento do desemprego, degradação de postos de trabalho e insegurança quanto à estabilidade no emprego (PEREIRA, 2006).

Diante deste cenário mundial, que atingiu o Brasil, inclusive as indústrias baianas, os trabalhadores se depararam com ameaças a suas vidas ocupacional, social e econômica, levando a preocupações e sentimentos de perdas, o que pode ter afetado a saúde mental destes indivíduos. Estes se afligiram com a possibilidade de perder o emprego (que é visto como uma espécie de "catalisador social", muitas vezes, e provedor da sobrevivência), o que provocaria mudanças nas condições de vida e de trabalho (LINHART, 2009; FRANCO; DRUCK; SELIGMANN-SILVA, 2010).

Nesta conjuntura econômica, onde a instabilidade no emprego era realidade para uma classe de trabalhadores que tinha a estabilidade garantida, observou-se que a prevalência de TMC foi maior entre os homens do que entre as mulheres. Isto pode ser explicado pelo fato da mão-de-obra masculina, além de ser maioria na indústria brasileira, conforme dados do IBGE (2010), ainda apresentar uma relação com o trabalho de não apenas sobrevivência, mas também de auto-afirmação na sociedade e provedor 
da família. A suspeita da perda deste trabalho, acabou propiciando sentimentos de angústia, de medo, de sofrimento, o que pode ter favorecido uma prevalência elevada destes transtornos (REBOUÇAS, LEGAY; ABELHA, 2007; POCHMANN, 2009).

Os trabalhadores mais jovens e com renda média mensal elevada foram os que apresentaram as maiores prevalências de transtornos mentais comuns. Durante o momento da inflexão do ritmo de expansão da economia, o funcionamento desfavorável do mercado de trabalho, o desemprego, além da rotatividade da mão-de-obra no setor industrial, estavam mais concentrados entre os trabalhadores mais jovens e aqueles que eram melhores remunerados. Entre os mais jovens, a falta de experiência, de recursos financeiros e de uma rede de contatos ampla favoreceram a instabilidade e rotatividade no mercado, além do medo e da incerteza do prejuízo sobre os projetos de desenvolvimento profissional e pessoal. E entre aqueles indivíduos com remunerações mais altas, demitia-se mais em troca das contratações de outros trabalhadores em condições inferiores de salário (POCHMAN, 2009; SHILTON, 2010; SPITZ, 2010).

Quanto à situação conjugal, concordando com outros estudos, a maior prevalência de TMC foi observada entre os trabalhadores divorciados/viúvos. Este achado pode ser explicado devido ao fato desses trabalhadores não disfrutarem de vínculos afetivos estabelecidos pela convivência familiar, a qual propicia apoio para enfrentar melhor as dificuldades no ambiente de trabalho., já que este encontra em sua família fontes de apoio para enfrentar melhor alguma dificuldade no ambiente de trabalho (LIPP; TANGANELLI, 2002; DIENER, 2000; LOPES; FAERSTEIN; CHOR, 2003).

A prevalência de TMC também foi maior entre os trabalhadores com nível de escolaridade mais elevado, e isto se deve provavelmente, ao fato da mãode-obra industrial, com nível superior, corresponder àquela com maiores salários e que sofreu com a insegurança das demissões, para se contratar uma mãode-obra com menor custo, favorecendo o sofrimento psíquico destes trabalhadores.

Com relação às variáveis ocupacionais, as maiores prevalências de TMC foram observadas entre os indivíduos que trabalhavam acima de 40 horas e acima de 6 dias, por semana e pelo menos um dia durante a noite. Trabalhadores com carga horária e dias de trabalho elevados, acabam sendo expostos por mais tempo as demandas físicas e cognitivas exigidas, ao ritmo de produção intenso, e a outros fatores que contribuam para o sofrimento mental. Quanto ao trabalho em turno noturno, pesquisas revelam que este se mostra mais nocivo à saúde mental, já que o organismo humano está adaptado ao trabalho diurno e ao descanso e reconstituição das energias a noite (GUIMARÃES, TEIXEIRA, 2003; BORGES, 2005; FERNANDES; ASSUNÇÃO; CARVALHO, 2010).

$\mathrm{Na}$ associação bruta dos grupos ocupacionais com os transtornos mentais comuns, o grupo com a menor prevalência de transtornos mentais comuns foi o dos técnicos de nível médio (GG3). Este grupo é formado por profissionais que necessitam ter nível médio completo mais curso profissionalizante. As tarefas realizadas por eles são basicamente de planejamento, execução e controle de normas e procedimentos, a fim de assegurar a qualidade das atividades. A menor prevalência de TMC neste grupo, pode ser devido a uma maior autonomia para praticar e desenvolver suas competências e maior controle sobre seu processo de trabalho, proporcionando o uso de suas habilidades e tomada de decisão de maneira mais atuante, o que, em conjunto com uma possível baixa tensão cognitiva dos processos de trabalho, pode favorecer a diminuição do risco de exigências psicológicas e o adoecimento psíquico (MORIN, 2001; BRASIL, 2002; HOSMER; LEMESHOW; MAY, 2008; SOUZA et al., 2010).

O grande grupo ocupacional com a maior prevalência de TMC foi o dos trabalhadores de serviços administrativos (GG4). Este grupo é formado por trabalhadores que realizam trabalhos burocráticos, sem contato constante com o público, que não necessitam de um nível elevado de escolaridade. Este achado provavelmente pode ser explicado em virtude do caráter operacional que este grupo apresenta, com baixo nível de autonomia para organizar sua forma de trabalhar, estabelecer suas normas e regras, o que pode contribuir para a condução de desajustes motivacionais influindo na saúde mental do indivíduo. Além disso, outro fator que pode justificar esta prevalência é o fato que, mesmo se tratando de trabalhadores com empregos com vínculos formais, estes podem ser caracterizados por limitados benefícios, insegurança no trabalho, curto tempo de manutenção do emprego e baixos salários (BRASIL, 2002; ARAÚJO; GRAÇA; ARAÚJO, 2003, BORGES, 2005, FERNANDES; ASSUNÇÃO; CARVALHO, 2010).

O segundo grupo ocupacional com a maior prevalência de TMC foi o dos trabalhadores agropecuários, florestais da caça e da pesca (GG6). Este grupo engloba os trabalhadores com nível fundamental, que desempenham atividades no trabalho com elevada exigência física, estando em geral, em posições desconfortáveis por longos períodos de trabalho. O grupo apresentou uma prevalência relativamente alta, comparada ao grupo de referência (GG3), provavelmente por se tratar de mão-de-obra pouco qualificada, associada a alta exigência 
física e mental proveniente das pressões dos superiores dentro do binômio aumento de produção em menor tempo e aumento do ritmo de trabalho (FERNANDES; ASSUNÇÃO; CARVALHO, 2010).

Outro ponto que pode estar relacionado às maiores prevalências destes transtornos mentais comuns nestes dois grupos é a "precarização subjetiva". Esta é uma expressão que afeta os trabalhadores assalariados com empregos estáveis, confrontados com demandas cada vez maiores no ambiente de trabalho e que estão preocupados com o fato de nem sempre estar em condições de atendê-las. Isto pode contribuir para o sofrimento mental, já que mantêm estes trabalhadores em um estado de insegurança, pelo medo de não serem capazes de atingir e cumprir as exigências de seus superiores (LINHART, 2009).

No início do estudo, imaginou-se que o grupo que apresentaria a maior prevalência de TMC seria o GG7, o qual concentra os trabalhadores da produção extrativa e da construção civil, que mobilizam habilidades psicomotoras e mentais voltadas, sobretudo, à forma do produto. Este grupo, apesar de ter tarefas desempenhadas com altas exigências físicas e cognitivas, em ritmo temporal intenso e baixa autonomia, pode ter apresentado uma prevalência não tão elevada, devido ao desenvolvimento de estratégias e de mecanismos de adaptação às circunstâncias adversas que favoreceram a saúde mental. Além disso, este grupo contém os trabalhadores da construção civil, cujo setor, mesmo com a crise mundial, obteve, na Bahia, elevado crescimento. A demanda por esta mão-de-obra pode ter contribuído para que estes profissionais, mesmo estando sobre tensão física e mental, compensassem estas exigências por meio da satisfação da empregabilidade e estabilidade adquiridas em decorrência do crescimento do setor (LIPP; TANGANELLI, 2002; BRASIL, 2002; SANTANA; OLIVEIRA, 2004).

Após o ajustamento do modelo estatístico pelas co-variáveis do estudo, verificou-se que as associações entre transtornos mentais comuns e os grupos ocupacionais se mantiveram estatisticamente significantes, com aumento nas razões de prevalências dos grupos, GG1 (organizações de interesse público e de empresas e gerentes) e e GG2 (profissionais das ciências e das artes).

O grupo GG1 é formado por ocupações que desempenham atividades de liderança, chefia. Para Brant e Dias (2004), o nível gerencial, sofre diariamente a pressão demanda - temporalidade, que dissocia a demanda do trabalho do tempo necessário para execução desta, tornando o tempo instantâneo, uma sucessão infindável de presentes, onde tudo deve ser realizado sob a pressão do agora, sendo que esta realidade seria fator favorável para desen- cadear nocividade à saúde mental. O grupo GG2 (na população deste estudo predominaram analistas e engenheiros) requer trabalhadores com alto nível de conhecimento profissional, cientifico e intelectual, e que no processo de trabalho desenvolvem produtos, administram, coordenam, controlam e analisam (BRASIL, 2002).

Os profissionais destes dois grupos vivem a realidade dicotômica da exigência de serem profissionais altamente flexíveis (abertos às mudanças de curto prazo e que tomam decisões rápidas) e da obediência às condições impostas pela organização do trabalho. Além disso, fatores como competitividade maximizada, cumprimento de metas em menor tempo, capacidade de mudança e inovação continuada e excelência no trabalho, em que idéias de perfeição e superioridade contribuem para a coerção à perfeição humana, na medida em que ignoram os limites dos processos fisiológicos e mentais, geram ansiedade e angústia diante da possibilidade de não conseguir atender às exigências e necessidades da empresa. Além disso, o cenário de incerteza econômica do momento, gerando medo sobre o futuro profissional e sentimento de não estar protegido, pode ter contribuído para esta elevada prevalência de TMC nestes dois grupos (BRANT; DIAS, 2004; FRANCO; DRUCK; SELIGMANN-SILVA, 2010).

Quanto às limitações deste trabalho, o presente estudo incluiu todos os industriários vinculados na base de dados do Serviço Social da Indústria, na Bahia, em 2009, referindo-se a uma população de indivíduos e não a uma amostra de trabalhadores. Por se tratar de uma população, a presença de erros sistemáticos na seleção dos entrevistados foi reduzida. Mas alguns fatores limitantes merecem ser destacados.

Por ter sido um estudo de corte transversal, pode ter apresentado a limitação verificada através do viés de prevalência, já que, por revelar a imagem instantânea do que se deseja estudar, pode identificar apenas os sobreviventes ao efeito estudado e sua situação quanto à exposição presente. $\mathrm{O}$ que pode explicar a prevalência global de TMC mais baixa em relação a outros estudos ocupacionais, mas semelhante à prevalência na população geral (PASQUALI et al., 1996; MEDRONHO; BLOCH, 2008).

Outro fator limitante foi a falta de estudos sobre transtornos mentais comuns em industriários, sobretudo utilizando o instrumento de rastreabilidade destes transtornos, o GHQ-12. Além disso, pelo fato do GHQ-12 ser um instrumento de rastreamento, ele é sensível a mudanças recentes no estado psicológico dos indivíduos, não permitindo a distinção daqueles com um diagnóstico psiquiátrico estabelecido. Foram encontrados estudos envolvendo outros instrumentos, como o SRQ - 20, por exemplo, o que impediu uma melhor comparabilidade entre os re- 
sultados obtidos, já que os mesmos analisam variáveis distintas, apresentam escalas e pontos de cortes diferentes (GOLDBERG; HUXLEY, 1992; PASQUALI et al., 1996)

Dificuldades também foram encontradas durante a busca de estudos que envolvessem os transtornos mentais comuns, a indústria e os grupos ocupacionais. A maioria dos estudos nesta temática é referente a ocupações não industriais, o que limitou a comparação dos achados aqui encontrados com os de outras pesquisas.

Como se tratou de um diagnóstico realizado por uma instituição nacional, que buscou de início verificar a situação de saúde dos trabalhadores da indústria, com enfoque a outras doenças crônicas, não sendo objetivo principal a análise da saúde mental, percebeu-se que o questionário utilizado nas entrevistas não abordou de maneira mais profunda dados sobre a organização, as condições e o conteúdo do trabalho, o que levou a uma não comparabilidade destes fatores sobre a saúde mental destes indivíduos, de maneira mais complexa.

Fatores como estes tem influenciado o aparecimento do sofrimento psíquico nos grupos ocupacionais, como têm demonstrado estudos relacionados a saúde mental realizados por diversos pesquisadores. Os fatores psicossociais do trabalho, por exemplo, têm sido estudados e tem se verificado a grande influência que estes exercem sobre o aparecimento do sofrimento mental em várias populações ocupacionais, contudo não foram contemplados no questionário, o que impediu se verificar a relação destes com a prevalência dos transtornos mentais (ARAÚJO; GRAÇA; ARAÚJO, 2003; ARAÚJO et al., 2003; SOUZA et al., 2010).

Este estudo permitiu observar que as ocupações têm um papel relevante no estado de saúde mental dos trabalhadores, havendo a necessidade de se desenvolverem mais estudos aprofundando a análise, com a inserção de outras variáveis relacionadas às condições e organização do trabalho de cada grupo ocupacional.

Verificou-se também que o instrumento utilizado para o rastreamento dos TMC, foi adequado, apesar das diversas limitações do próprio estudo e de se tratar de uma característica subjetiva, como a saúde mental;

Como sugestões para outros trabalhos envolvendo a população industrial, ressaltam-se a importância de se investigar quais os fatores de maior impacto em relação aos TMC e quais alternativas podem ser criadas para evitá-los, minimizá-los e/ou tratá-los, no ambiente ocupacional.

\section{Considerações finais}

Este estudo permite concluir que as questões relativas à saúde mental e ocupações entre os industriários são muito insipientes, apresentando um leque de possibilidades de investigações para implementação de ações que venham a promover e prevenir a saúde mental dos trabalhadores deste setor da economia brasileira.

Sendo assim, faz-se necessário:

- Realizar estudos futuros englobando as características psicossociais do trabalho na indústria, além das condições, organização e processo do trabalho de acordo com os grupos ocupacionais;

- Levantar dados sobre o conhecimento dos profissionais de saúde e segurança quanto aos fatores de risco psicossociais, e proporcionar ações de intervenção para o preparo dos mesmos frente à saúde mental destes trabalhadores;

- Realizar futuros estudos que possibilitem a avaliação dos impactos dos fatores de risco sobre o desempenho social e ocupacional do trabalhador e como isto pode contribuir para a melhoria da indústria;

- Verificar a possibilidade de realização de monitoramento dos fatores de risco destes transtornos mentais e da criação de estratégias que minimizem ou evitem o comprometimento da saúde mental.

O estudo aponta também para a necessidade da ampliação da discussão desta temática entre os grupos ocupacionais constantes na CBO, já que a dificuldade em se encontrar estudos semelhantes para comparação foi um fator limitante para a discussão.

A partir deste estudo, se propõe também a elaboração de uma pesquisa de base nacional, entre os industriários brasileiros, sobre a saúde mental e os aspectos psicossociais, a fim de se obter um banco único, com informações que respaldem a criação de estratégias especificas de prevenção e promoção da saúde psíquica dos trabalhadores da indústria brasileira, de acordo com as peculiaridades de cada grande grupo ocupacional.

\section{Agradecimento}

As autoras agradecem a contribuição do SESI/DR/BA por disponibilizar os dados da pesquisa para realização do presente trabalho. 


\section{Contribuições de autoria}

Fonseca, I. S. S.: Contribuição substancial no projeto e delineamento, no levantamento de dados e na sua análise e interpretação, além da elaboração do manuscrito e revisão crítica. Araújo, T. M.: Elaboração e revisão crítica do manuscrito e aprovação final da versão a ser publicada.

\section{Referências}

ARAÚJO, T. M. et al. Saúde e trabalho docente: dando visibilidade aos processos de desgaste e adoecimento docente a partir da construção de uma rede de produção coletiva, Educação em Revista, Belo Horizonte, v. 37, p. 183-212, 2003.

ARAUJO, T. M.; GRAÇA, C. C.; ARAUJO, E. Estress ocupacional e saúde: contribuições do modelo demanda-controle. Ciência \& Saúde Coletiva, Rio de Janeiro, v. 8, n. 3, p. 285-297, 2003.

BENAVIDES, F. G. et al. Descripción de los factores de riesgo psicosocial em cuatro empresas. Gaceta Sanitaria, Barcelona, v. 16, n. 3, p. 222-229, 2002.

BORGES, E. M. Saúde mental, trabalho em turnos e qualidade do sono de funcionários da linha de produção de uma indústria moveleira de Arapongas, PR. 2005. 112 f. Dissertação (Mestrado em Psicologia)-Universidade Católica Dom Bosco, Campo Grande, 2005.

BOUYER, G. C. Contribuição da Psicodinâmica do Trabalho para o debate: "o mundo contemporâneo do trabalho e a saúde mental do trabalhador", Revista Brasileira de Saúde Ocupacional, São Paulo, v. 35, n. 122, p. 249-259, 2010.

BRANT, L. C.; DIAS, E. C. Trabalho e sofrimento em gestores de uma empresa pública em reestruturação. Cadernos de Saúde Pública, Rio de Janeiro, v. 20, n. 4, p. 942-949, jul./ago., 2004.

BRASIL. Ministério do Trabalho e Emprego. Classificação Brasileira de Ocupações - CBO, 2002. Brasília, DF: Ministério do Trabalho e Emprego, 2002. Disponível em: <http://www.mtecbo.gov.br/cbosite/ pages/home.jsf $>$. Acesso em: 01 fev. 2010.

BRASIL. Ministério da Saúde. Resolução n. 196/96. Diretrizes e normas regulamentadoras de pesquisa envolvendo seres humanos. Brasília, DF: Ministério da Saúde, 1996.

COUTINHO, L. M. S.; SCAZUFCA, M.; MENEZES, P. R. Métodos para estimar razão de prevalência em estudos de corte transversal. Revista de Saúde Pública, São Paulo, v. 42, n. 6, p. 992-998, 2008. Disponível em: <http://www.scielosp.org/pdf/rsp/v42n6/7118. pdf $>$. Acesso em: 07 jan. 2013.

DIENER, E. Subjective well-being: The science of happiness and a proposal for a national index. American Psychologist, Washington, v. 55, n. 1, p. 3443, 2000.
FEDERAÇÃO DAS INDÚSTRIAS DO ESTADO DA BAHIA. Guia Industrial do Estado da Bahia, 2010. Salvador: Fieb, 2010. Disponível em: <http://www. fieb.org.br/guia/default.asp>. Acesso em: 20 abr. 2010. FEDERAÇÃO DAS INDÚSTRIAS DO ESTADO DA BAHIA. Número de indústrias que procuram a Bahia cresce 88\% em 2009. Salvador: Fieb, 2009. Disponível em: <http://adm.agecom.ba.gov.br/conteudo/ noticias/2009/08/17/numero-de-industrias-queprocuram-a-bahia-cresce-88-em-2009/base_view $>$. Acesso em: 14 jan. 2010.

FERNANDES, R. C.; ASSUNÇÃO, A. A.; CARVALHO, F. M. Mudanças nas formas de produção na indústria e a saúde dos trabalhadores. Ciência e Saúde Coletiva [online], Rio de Janeiro, v. 15, suppl. 1, p. 1563-1574, 2010. Disponível em: <http://www.scielo.br/pdf/csc/ v15s1/068.pdf>. Acesso em: 01 mar. 2010.

FONSECA, S. A. et al. Percepção de saúde e fatores associados em industriários de Santa Catarina, Brasil. Cadernos de Saúde Pública, Rio de Janeiro, v. 24, n. 3, p. 567-576, mar. 2008.

FRANCO, T.; DRUCK, G.; SELIGMANN-SILVA, E. As novas relações de trabalho, o desgaste mental do trabalhador e os transtornos mentais no trabalho precarizado. Revista Brasileira de Saúde Ocupacional, São Paulo, v. 35, n. 122, p. 229-248, 2010.

GOLDBERG, D.; HUXLEY, P. Common mental disorders: a bio-social-model. London: Tavistock, 1992.

GOUVEIA, V. V. et al. A utilização do QSG-12 na população geral: estudo de sua validade de construto. Psicologia: Teoria e Pesquisa, Brasília, v. 19, n. 3, p. 241-248, set./dez. 2003.

GUIMARAES, L. A. M.; TEIXEIRA, L. N. Transtornos mentais e trabalho em turnos alternados em operários de mineração de ferro em Itabira (MG). Jornal Brasileiro de Psiquiatria, Rio de Janeiro, v. 52, n. 4, p. 283-289, 2003.

HÖFELMANN, D. A.; BLANK, N. Auto-avaliação de saúde entre trabalhadores de uma indústria no sul do Brasil. Revista de Saúde Pública, Rio de Janeiro, v. 41, n. 5, p. 777-787, 2007.

HOSMER, D. W.; LEMESHOW, S. Applied Logistic Regression. New York: John Wiley \& Sons, 2000.

HOSMER, D. W.; LEMESHOW, S.; MAY, S. Applied survival analysis: regression modeling of time-to-event data. $2^{\text {nd. }}$ edition. New Jersey: Wiley, 2008. 
INSTITUTO BRASILEIRO DE GEOGRAFIA E ESTATÍSTICA. Em 2009, expectativa de vida ao nascer era de 73, 17. IBGE, 2010. Disponível em: <http:// www.ibge.gov.br/home/presidencia/noticias/noticia visualiza.pph >. Acesso em: 01 dez. 2010.

KLEINBAUM, D. G.; KLEIN, M. Logistic regression: a self-learning text. $2^{\text {nd }}$ edition. New York: SpringerVerlag, 2002.

LINHART, D. Modernisation et précarisation de la vie au travail, Papeles del CEIC, n. 43, 2009. Disponível em: <http://www.identidadcolectiva.es/pdf/43.pdf> Acesso em: 01 mar. 2010.

LIPP, M. E. N.; TANGANELLI, M. S. Stress e qualidade de vida em magistrados da justiça do trabalho: diferenças entre homens e mulheres. Psicologia: Reflexão e Crítica, Porto Alegre, v. 15, n. 3, p. 537-548, 2002. Disponível em: <http://www.scielo.br/pdf/prc/ v15n3/a08v15n3.pdf> . Acesso em: 19 dez. 2012.

LOPES, C. S.; FAERSTEIN, E.; CHOR, D. Eventos de vida produtores de estresse e transtornos mentais comuns: resultados do Estudo Pró-Saúde. Cadernos de Saúde Pública, Rio de Janeiro, v. 19, n. 6, dez. 2003. Disponível em: <http://www.scielo.br/scielo. php?script $=$ sci_arttext $\&$ pid $=$ S0102-311X2003000600 015\&lng $=$ pt\&nrm $=$ iso $>$. Acesso em: 02 mar. 2010.

LUDERMIR, A. B.; MELO FILHO, D. A. Saúde mental, condições de vida e estrutura ocupacional. Revista de Saúde Pública, Rio de Janeiro, v. 36, n. 2, p. 213-231, 2002.

MEDRONHO, R. A.; BLOCH, K. V. Epidemiologia. 2. ed. São Paulo: Atheneu. 2008.

MOLINA, A. S.; MIASSO, A. I. Benzodiazepine use among employees of a private company. Revista Latino-Americana de Enfermagem, Ribeirão Preto, v. 16, p. 517-522, jul./ago., 2008. Disponível em: <www. eerp.usp.br/rlae >. Acesso em: 06 fev. 2011.

MORIN, E. M. Os sentidos do trabalho. Revista de Administração de Empresas, São Paulo, v. 41, n. 3, p. 8-10, jul./set., 2001

NAHAS, M. V. Atividade física, saúde e qualidade de vida: conceitos e sugestões para um estilo de vida ativo. 2. ed. Londrina: Midiograf, 2001.
ORGANIZACIÓN MUNDIAL DE LA SALUD. Que és la salud mental? 2007. Disponível em: <http://www. who.int/features/qa/62/es/index.html>. Acesso em: 06 fev. 2011.

PAN AMERICAN HEALTH ORGANIZATION - PAHO. Reforma de La salud mental: alcohol y el peso de lãs decisiones. 2001. Disponível em: < http://www.paho. org/Spanish/DD/PIN/saludmental_007.htm>. Acesso em: 06 fev. 2011.

PASQUALI, L. et al. QSG - Questionário de Saúde Geral de Goldberg: manual técnico QSG. São Paulo: Casa do Psicológo, 1996.

PEREIRA, M. G. Epidemiologia: teoria e prática. Rio de Janeiro: Guanabara Koogan, 2006.

POCHMANN, M. O trabalho na crise econômica no Brasil: primeiros sinais. Estudos Avançados, São Paulo, v. 23, n. 66, p. 41-52, 2009. Disponível em: <http://www.scielo.br/scielo.php?script=sci_arttext \&pid $=$ S0103-40142009000200004 > . Acesso em: 01 mar. 2010.

REBOUÇAS, D.; LEGAY, L. F.; ABELHA, L. Satisfação com o trabalho e impacto causado nos profissionais de serviço de saúde mental. Revista de Saúde Pública, Rio de Janeiro, v. 41, n. 2, p. 244-250, 2007.

SANTANA, V.; OLIVEIRA, R. P. Saúde e trabalho na construção civil em uma área urbana do Brasil, Cadernos de Saúde Pública, Rio de Janeiro, v. 20, n. 3, p. 797-811, maio/jun. 2004.

SHILTON, J. Desemprego mundial entre os jovens atinge níveis recordes. World Socialist Web Site. 2010. Disponível em: <http://www.wsws.org/ pt/2010/sep2010/por1-s17.shtml>. Acesso em: 06 mar. 2010.

SOUZA, S. F. Fatores psicossociais do trabalho e transtornos mentais comuns em eletricitários. Revista de Saúde Pública, São Paulo, v. 44, n. 4, p. 710-717, 2010.

SPITZ, C. Crise econômica faz desemprego mundial entre jovens ter alta recorde. O Globo. 2010.

Disponível em: <http://oglobo.globo.com/economia/ mat/2010/08/11/crise-economica-faz-desempregomundial-entre-jovens-ter-alta-recorde-917373280. asp2010>. Acesso em: 06 mar. 2010. 